\title{
Paramètres génétiques de la concentration plasmatique en FSH des agnelles de race Lacaune viande
}

\author{
L. BODIN, B. BIBE, M. BLANC (*), G. RICORDEAU \\ avec la collaboration de R. Mollaret et J.C. Poirier (*) \\ I.N.R.A., Station d'Amélioration Génétique des Animaux, \\ Centre de Recherches de Toulouse, Auzeville, \\ B.P. 27, F 31326 Castanet Tolosan Cedex \\ (*) I.N.R.A., Station de Physiologie de la Reproduction \\ Centre de Recherches de Tours, F 37380 Nouzilly
}

\begin{abstract}
Résumé
Cette étude estime la variabilité phénotypique et génétique des variables FSH et $\log$ FSH mesurées à partir d'un seul prélèvement sanguin aux environs de 33 jours, sur 892 agnelles Lacaune viande nées de juillet à septembre 1983 dans 21 élevages et issues de 42 pères utilisés en insémination. Les effets " élevage » et " père " sont très significatifs. L'héritabilité est de 0,38 pour le taux de FSH et de 0,47 pour le log FSH, avec des variances génétiques père et mère équivalentes. Les effets "type de naissance " et "gain 10-30 jours" ne sont pas significatifs. L'effet " heure de prélèvement » est significatif uniquement pour le log FSH. Le taux de FSH des agnelles pourrait donc être utilisé comme critère de sélection, si les études ultérieures confirment son intérêt comme indicateur précoce de la prolificité.
\end{abstract}

Mots clés : Brebis, race Lacaune viande, plasma FSH avant la puberté, héritabilité.

\section{Summary \\ Genetic parameters of plasma FSH level of Lacaune meat ewe-lambs}

This study estimates the phenotypic and genetic variability of plasma FSH level (FSH and log FSH) measured from a single blood sample collected at approximately 33 days of age, from 892 ewe-lambs born in July-September 1983, in 21 flocks, from 42 A.I. sires. The flock and sire effects were highly significant. Heritability was 0.38 and 0.47 for plasma FSH and log plasma FSH, with an equivalent sire, and dam genetic variance. The effects of litter size at birth and growth from 10 to 30 days were not significant. The effect of sampling time was significant for log FSH. The plasma FSH level of ewe-lambs could then be used as criterion of selection, if the future studies confirm its interest as an early indicator of prolificacy.

Key words : Ewes, Lacaune meat breed, prepubertal plasma FSH, heritability. 


\section{Introduction}

Les observations de Findlay \& Bindon (1976), RicordeAu et al. (1984), Bindon et al. (1985), semblent indiquer que le taux plasmatique de FSH des agnelles de 3 à 6 semaines pourrait constituer un critère précoce de leur prolificité. Cependant ces résultats ont été obtenus sur des effectifs limités et en comparant des agnelles de types génétiques différents. Il importait donc d'entreprendre des études complémentaires en race pure et en fermes pour estimer les paramètres génétiques de ce critère précoce et mesurer de façon plus précise son intérêt pour la sélection.

Cette étude a pour but d'estimer l'héritabilité du taux de FSH des agnelles de race Lacaune viande dans le cadre du programme de sélection conduit par la Coopérative Ovitest sur la prolificité en rythme accéléré de reproduction. Pour avoir une estimation suffisamment précise, nous avons choisi d'effectuer un seul contrôle sur un maximum d'agnelles, le stade optimum du prélèvement ayant été déterminé lors d'une étude préliminaire ${ }^{(1)}$. Ce protocole a été largement facilité par le fait que les agnelles sont nées de mères inséminées sur astrus synchronisé.

\section{Matériel et méthodes}

\section{A. Protocole et conditions d'élevage}

De février à avril 1983, la semence de 42 béliers a été utilisée dans 21 élevages, ce qui a permis d'obtenir 892 agnelles contrôlées. Chaque descendance compte en moyenne 21 filles réparties dans 9 élevages, de sorte que chaque élevage possède des filles de 4 à 5 pères.

Les agnelles se répartissent en 3 types de naissance : 23 p. 100 de simples, 52 p. 100 de doubles et 25 p. 100 de multiples (3 et plus), ce qui correspond à une prolificité des mères de 1,75. Elles sont élevées en bergerie et allaitées presque uniquement sous la mère, avec allaitement artificiel en complément pour les multiples. Leur mise à la reproduction a lieu à partir de 11 mois.

\section{B. Mesures et dosages}

Un seul échantillon de sang est prélevé au niveau de la veine jugulaire, sur chacune des 892 agnelles. Dans chaque élevage, la prise de sang a lieu le même jour, 5 semaines après la naissance du premier agneau, de sorte que l'on observe 2 classes d'âge à la collecte : 29 à 41 jours (moyenne 34 jours) pour 92 p. 100 des agnelles nées des fécondations sur œstrus induit, et 12 à 21 jours (moyenne 17 jours) pour les $8 \mathrm{p}$. 100 nées des fécondations sur « retour».

(1) L'étude préliminaire, effectuée sur 66 agnelles Lacaune nées en mars-avril 1983 et contrôlées 3 fois à 21 jours d'intervalle, a permis de constater que le taux de FSH est légèrement plus élevé à 5 semaines, mais ne varie pas de façon significative de 2 à 7 semaines (entre 4,8 et $5,0 \mathrm{ng} / \mathrm{ml}$ ). 
Les prélèvements sanguins ont lieu au cours de la journée. L'heure de prélèvement est classée suivant 4 périodes : $8 \mathrm{~h}$ à $10 \mathrm{~h} ; 10 \mathrm{~h}$ à $12 \mathrm{~h} ; 14 \mathrm{~h}$ à $16 \mathrm{~h}$ et $16 \mathrm{~h}$ à $18 \mathrm{~h}$. Les échantillons $(5 \mathrm{ml})$ sont prélevés dans des tubes héparinés et centrifugés dans chaque élevage à la fin de la collecte. Après pipetage, le plasma est congelé sur place à $-12^{\circ} \mathrm{C}$.

Pour les 13 élevages en contrôle de croissance, la collecte des échantillons est effectuée en même temps qu'une des pesées du contrôle réalisé tous les 21 jours, ce qui permet de calculer le gain moyen quotidien des agneaux de 10 à 30 jours (gain 10-30 jours).

La concentration en FSH est mesurée par dosage radio-immunologique homologue selon la méthode décrite par BLANC \& PoIRIER (1979) et exprimée en ng de FSH, HG225 par ml de plasma. Tous les échantillons sont analysés en double et en même temps, de manière à minimiser la variabilité des résultats. La prise d'essai est de $100 \mu \mathrm{l}$ pour tous les échantillons, ce qui donne en moyenne, pour $7,7 \mathrm{ng} / \mathrm{ml}$, une valeur de $\mathrm{B} /$ $\mathrm{B}_{\mathrm{o}}$ de 50 p. $100^{(2)}$. Pour les valeurs de $13-17,7-8$ et $3,5-4,5 \mathrm{ng} / \mathrm{ml}$, le coefficient de variation (C.V.) est de 10,1-8,9 et 12,6 p. 100 respectivement (C.V. moyen calculé à partir de 50 duplicata d'échantillons de chaque classe).

\section{Variables}

Les variables analysées sont la concentration plasmatique en FSH et sa transformée logarithmique. D'autres transformations ont été utilisées $\left[x^{y}\right.$ et $\left.\log (x+\lambda)\right]$, mais seule la transformation $\log \mathrm{x}$ permet d'obtenir la symétrie de la distribution des résidus, l'aplatissement de la loi normale et la quasi-normalité.

\section{Analyses statistiques}

Nous avons tout d'abord mesuré l'effet possible des différents facteurs de variation sur la FSH et sa transformée logarithmique à l'aide d'un modèle d'analyse de variance à effets fixes, avec l'heure de prélèvement, l'élevage intra heure, l'âge de l'agnelle et le mode de naissance, considérés comme facteurs. De manière à minimiser les biais de ces estimations, l'effet du père est pris en compte dans ce modèle. Sur l'échantillon réduit des élevages en contrôle de croissance, nous avons complété ce modèle par l'addition du gain 10-30 jours de l'agnelle en covariable :

$Y^{t}=X P^{t}+Z \beta^{t}+b^{t} C+E^{t}$

$\mathrm{Y}^{\mathrm{t}}$ : vecteur des observations de dimension $\mathrm{n}$ pour la variable $\mathrm{FSH}(\mathrm{t}=1)$ et sa transformée logarithmique $(\mathrm{t}=2)$.

$P^{1}$ : vecteur des valeurs des pères, de dimension $\mathrm{p}$.

$\beta^{\prime}$ : vecteur d'effets fixes ( $y$ compris la moyenne $\mu$ ) de dimension $f$.

$\mathrm{C}$ : vecteur des gains $10-30 \mathrm{j}$, de dimension $\mathrm{n}$.

$E^{t}$ : vecteur des variables résiduelles, de dimension $n$.

(2) $\mathrm{B}=$ quantité de radioactivité liée à l'anticorps pour l'échantillon considéré.

$B_{\mathrm{v}}=$ quantité de radioactivité liée à l'anticorps en l'absence d'hormone froide. 
$X$ : matrice de dimension $(n, p)$ décrivant l'incidence des pères pour chaque observation.

$\mathrm{Z}$ : matrice de dimension $(n, f)$ décrivant l'incidence des effets fixes pour chaque observation.

$b^{\prime}$ : coefficient de régression des variables sur le gain $10-30 \mathrm{j}$.

Nous avons ensuite calculé l'héritabilité, à partir des composantes de la variance père $\left(\sigma_{p}^{2}\right)$ et mère $\left(\sigma_{m}^{2}\right)$ estimées par 2 modèles hiérarchiques aléatoires, l'un tenant compte uniquement du facteur père (modèle 1), l'autre du facteur père et du facteur mère hiérarchisé sur père (modèle 2).

\section{Résultats}

La concentration de FSH plasmatique est très variable (valeur moyenne de $7,4 \mathrm{ng}$, écart type de $4,8 \mathrm{ng}$ ) mais la distribution est très dissymétrique (variation de 0,8 à 43,2 ng). La transformation logarithmique permet de rétablir la symétrie de la distribution des résidus ( $b_{1}$ de Pearson respectivement de 10,7 et 0,31 pour $\mathrm{FSH}$ et $\log \mathrm{FSH}$ ) et d'en augmenter son aplatissement $\left(b_{2}\right.$ de Pearson respectivement de 18,0 et 4,6 pour FSH et $\log$ FSH) ${ }^{(3)}$. Notons cependant que cette transformation ne permet pas d'obtenir la normalité des résidus.

La covariable «gain $10-30 \mathrm{j}$ » ne s'est révélée significative pour aucune des 2 variables étudiées, aussi avons-nous choisi de présenter les résultats de l'analyse de variance concernant les effets fixes obtenus à partir du modèle sans covariable, mais portant sur la totalité des données (tabl. 1). De cette analyse, il ressort que les effets "âge à la mesure " et "type de naissance des agnelles ", ne sont jamais significatifs. L'effet « heure " n'est significatif que sur la variable $\log$ FSH, le niveau de FSH étant minimum de 10 à $14 \mathrm{~h}(7,1 \mathrm{ng})$ et maximum autour de $9 \mathrm{~h}$ et de $15 \mathrm{~h}$ (respectivement 7,7 et $7,6 \mathrm{ng}$ ). L'effet « élevage » est, par contre, très significatif quelle que soit la variable considérée.

Les résultats de l'analyse hiérarchique (tabl. 2) confirment l'existence d'un effet "père " très significatif, déjà mis en évidence dans le modèle d'étude des effets fixes. L'effet « mère » n'apparaît pas significatif, mais l'estimation de la composante de la variance due à la mère n'est pas sensiblement différente de l'estimation de celle due au père.

Les héritabilités sont de 0,38 pour le taux de FSH et de 0,47 pour la variable transformée log FSH.

(3) - Pour une loi normale, $b_{1}=0$ et $b_{2}=3$. 


\section{TABleau 1}

Estimation des effets fixes par analyse de variance.

Estimates of fixed effects by analysis of variance of plasma FSH and log plasma FSH.

\begin{tabular}{|c|c|c|c|c|c|}
\hline \multirow[b]{2}{*}{ Source de variation } & \multirow[b]{2}{*}{ d.d.1. } & \multicolumn{2}{|c|}{ FSH } & \multicolumn{2}{|c|}{$\log$ FSH } \\
\hline & & $\begin{array}{c}\text { Carré } \\
\text { moyen }\end{array}$ & Test $F$ & $\begin{array}{c}\text { Carré } \\
\text { moyen }\end{array}$ & Test $\mathbf{F}$ \\
\hline Totale & 891 & 22,96 & & 0,24 & \\
\hline Heure de prélèvement & 3 & 28,30 & $1,38 \mathrm{NS}$ & 0,74 & $3,55 \mathrm{~S}$ \\
\hline Elevage intra heure & 18 & 51,80 & $2,53 \mathrm{TS}$ & 0,63 & $3,04 \mathrm{TS}$ \\
\hline Père ........... & 41 & 52,92 & $2,58 \mathrm{TS}$ & 0,64 & $3,10 \mathrm{TS}$ \\
\hline Type de naissance & 2 & 2,95 & $0,14 \mathrm{NS}$ & 0,08 & 0,37 NS \\
\hline Age au prélèvement & 1 & 3,44 & $0,17 \mathrm{NS}$ & 0,02 & $0,09 \mathrm{NS}$ \\
\hline Résiduelle ....... & 826 & 20,48 & & 0,21 & \\
\hline
\end{tabular}

TS $=$ très significatif $(P<0,01) ; S=$ significatif $(P<0,05) ; N S=$ non significatif.

Différences entre élevages et entre pères en ce qui concerne les valeurs brutes du taux de FSH :

- entre élevages : moyenne $7,5 \mathrm{ng} / \mathrm{ml}$; de 5,6 à 11,3 ,

— entre pères : moyenne $7,2 \mathrm{ng} / \mathrm{ml}$; de 3,9 à 12,6 .

TABLEAU 2

Composantes de la variance pour les variables FSH et log FSH, sans ou avec les mères.

Components of variance for plasma FSH and log plasma FSH, without or with the mothers.

\begin{tabular}{|c|c|c|c|c|}
\hline \multirow{2}{*}{ Composantes variance et $h^{2}$} & \multicolumn{2}{|c|}{ FSH } & \multicolumn{2}{|c|}{$\log$ FSH } \\
\hline & Modèle 1 & Modèle 2 & Modèle 1 & Modèle 2 \\
\hline $\begin{array}{l}\text { Variance totale }=\sigma_{\mathrm{T}}^{2} \ldots \ldots \ldots \\
\text { F père } \ldots \ldots \ldots \\
\text { F mère } \ldots \ldots \ldots \\
\text { Variance père }: \sigma_{\mathrm{p}}^{2} \ldots \ldots \ldots \\
\text { Variance mère }: \boldsymbol{\sigma}_{\mathrm{m}}^{2} \ldots \ldots \ldots \\
\text { Variance résiduelle }: \boldsymbol{\sigma}_{c}^{2} \ldots \ldots \ldots\end{array}$ & $\begin{array}{l}22,96 \\
3,1 \text { TS } \\
- \\
2,13 \\
- \\
20,06\end{array}$ & $\begin{array}{l}22,96 \\
3,1 \text { TS } \\
1,2 \text { NS } \\
2,08 \\
2,74 \\
17,38\end{array}$ & $\begin{array}{c}0,2417 \\
3,7 \mathrm{TS} \\
- \\
0,0276 \\
- \\
0,2048\end{array}$ & $\begin{array}{l}0,2417 \\
3,8 \text { TS } \\
1,1 \text { NS } \\
0,0274 \\
0,0119 \\
0,1929\end{array}$ \\
\hline $\begin{array}{l}\text { Héritabilité }\left(h^{2}\right) \\
\text { Ecart type de } h^{2}\end{array}$ & $\begin{array}{l}0,39 \\
0,11\end{array}$ & $\begin{array}{l}0,38 \\
0,11\end{array}$ & $\begin{array}{l}0,47 \\
0,13\end{array}$ & $\begin{array}{l}0,47 \\
0,13\end{array}$ \\
\hline
\end{tabular}

Modèle 1 = facteur père uniquement.

Modèle 2 = facteur père et facteur mère hiérarchisé sur père.

\section{Discussion}

\section{A. Distributions et transformation}

La distribution des valeurs brutes de FSH plasmatiques confirme les résultats antérieurs sur la variabilité du taux de FSH chez les jeunes agnelles. Les très hautes valeurs observées (FSH >14 $\mathrm{ng}$ ) semblent bien traduire une variabilité biologique, car 
nous n'avons actuellement aucune raison de penser qu'elles correspondent à des agnelles ayant un mauvais fonctionnement ovarien. Par ailleurs, les erreurs de mesures sont réduites par la pratique du double dosage et la vérification de la concordance des résultats.

La transformation logarithmique rétablit la symétrie, mais n'assure pas la normalité, bien que celle-ci soit approchée. Cette transformation diminue aussi les liaisons moyenne-variance que l'on observe intra-père ( $r=0,84$ pour FSH et 0,47 pour $\log$ FSH), permettant ainsi d'approcher les conditions d'homoscédasticité.

D'une manière générale, la transformation logarithmique paraît donc indispensable pour l'analyse statistique. Il est bien entendu qu'ultérieurement, seule la variable la plus liée aux caractères de reproduction des femelles et à l'index de leur père sera prise en compte.

\section{B. Facteurs non génétiques}

L'absence d'effet du type de naissance et de la croissance initiale (donc du poids au moment du prélèvement) est en accord avec les résultats de RicordeAu et al. (1984). Ceci est à rapprocher de l'absence d'effets maternels, mise en évidence par la faible différence des variances génétiques père et mère.

L'évolution du taux de FSH au cours de la journée (marquée par une décroissance en cours de matinée et une croissance au cours de l'après-midi) n'est pas très significative. Une évolution un peu similaire a été observée par CogNiE et al. (1984) chez les brebis adultes en anœstrus saisonnier. Il convient donc d'approfondir la connaissance de ce rythme et peut-être d'en tenir compte de façon plus fine dans les études ultérieures.

Le seul effet non génétique très significatif est l'élevage. Comme la plupart des 21 troupeaux utilisent en partie - grâce à l'I.A. - les mêmes béliers depuis une dizaine d'années, on peut penser que les différences génétiques dues aux pères sont négligeables et que celles dues aux mères sont faibles. Les différences observées doivent donc s'expliquer par des différences de conditions de milieu. Parmi celles-ci, l'alimentation et particulièrement le niveau protéique de la ration - serait capable d'augmenter la sécrétion plasmatique de FSH, tout au moins sur adultes quelques jours avant l'œstrus (BRIEN et al., 1976 ; KNIGHT et al., 1981).

\section{Conclusion}

L'héritabilité du taux de FSH aux environs de 33 jours, à partir d'un seul prélèvement, est relativement élevée si on la compare aux estimations concernant les variables pondérales calculées habituellement dans les mêmes conditions. Cette première estimation a une signification importante, car elle est obtenue en fermes, sur des effectifs élevés, avec des variances génétiques père et mère équivalentes. Elle est de toute façon supérieure à celle du taux de $\mathrm{LH}\left(\mathrm{h}^{2}\right.$ inférieure à 0,10$)$ estimée par BindoN \& PIPER (1976) sur des jeunes mâles. S'il se vérifie que le niveau de FSH est un critère précoce de la prolificité des femelles, on pourra donc envisager une sélection rapide et 
efficace sur ce critère, mais uniquement à partir des jeunes agnelles. Il convient donc de poursuivre cette étude pour vérifier que les différences observées entre descendances correspondent à des différences de prolificité.

Reçu le 28 juin 1985.

Accepté le 18 septembre 1985.

\section{Remerciements}

Nous remercions : les Eleveurs Sélectionneurs du schéma Lacaune viande, les Responsables du Centre d'I.A. Ovitest et de l'E.D.E. de l'Aveyron, qui ont permis la réalisation du protocole concernant l'utilisation des béliers et la collecte des prélèvements de sang; $M$. Courot, qui a encouragé cette étude et B.M. Bindon, pour ses suggestions à la lecture du manuscrit.

\section{Références bibliographiques}

Bindon B.M., Piper L.R., 1976. Assessment of new and traditional techniques of selection for reproduction rate. In: Tomes G.J., RoBERTSON D.E., LightFoOd R.J. (éd.), Sheep Breeding, 387-401, Butterworths, London.

Bindon B.M., Piper L.R., Cummins L.J., O'Shea T., Hillard M.A., Findlay J.K., Robertson D.M., 1985. Reproductive endocrinology of prolific sheep studies of the Booroola Merino. In : LAND R.B., Robinson D.W. (éd.), Genetics of reproduction in sheep, 217-235, Butterworths, London.

Blanc M.R., Poirier J.C., 1979. A new homologous radioimmunoassay for ovine follicle stimulating hormone : development and characterization. Ann. Biol. Anim. Biochim. Biophys., 19, 1011-1026.

Brien F.D., Baxter R.W., Findlay J.K., Cumming I.A., 1976. Effect of lupin grain supplementation on ovulation rate and plasma follicle stimulating hormone (FSH) concentration in maiden and mature Merino ewes. Proc. Austr. Soc. Anim. Prod., 11, 237-240.

Cognie Y., Schirar A., Martinet J., Poulin N., Mirman B., 1984. Activité reproductrice et maitrise de l'ovulation chez la brebis. ges Journées de la Recherche Ovine et Caprine, I.N.R.A.-I.T.O.V.I.C., Paris, 5-6 décembre 1984. La reproduction chez les Ovins et les Caprins, 109-113.

Findlay J.K., Bindon B.M., 1976. Plasma FSH in Merino lambs selected for fecundity. J. Reprod. Fert., 46, 515-516 (Abstr.).

Knight T.W., Payne E., Peterson A.J., 1981. Effect of diet and live weight on FSH and ostradiol concentrations in Romney ewes. Proc. Aust. Soc. Repr. Biol., 19 (Abstr.).

Ricordeau G., Blanc M.R., Bodin L., 1984. Teneurs plasmatiques en FSH et LH des agneaux mâles et femelles issus de béliers Lacaune prolifiques et non prolifiques. Génét. Sél. Evol., 16 (2), 195-210. 